\title{
Distribution of Virulence Factors and Antimicrobial Resistance Properties of Uropathogenic Escherichia coli Isolated from Diabetic and Healthy Males Suffered from Urinary Tract Infections
}

\author{
Reza Rezaee $^{1}$, Amir Talebreza ${ }^{2}$, Katayoun Ziari ${ }^{3}$, \\ Vahid Behnod $^{4}$ and Bibi Fatemeh Shakhs Emampour ${ }^{5 *}$ \\ ${ }^{1}$ Ministry of Health and Medical Education, \\ Deputy of Curative Affaires Budget Administration, Tehran, Iran. \\ ${ }^{2}$ Department of Surgery , AJA University of Medical Sciences, Tehran, Iran. \\ ${ }^{3}$ Department of Pathology, Be'sat Hospital, AJA University of Medical Sciences, Tehran, Iran. \\ ${ }^{4}$ Baqiyatallah University of Medical Sciences, Tehran, Iran. \\ ${ }^{5}$ Department of Anesthesiology , Birjand University of Medical Sciences, Birjand, Iran.
}

http://dx.doi.org/10.13005/bbra/2117

(Received: 11 April 2016; accepted: 25 May 2016)

\begin{abstract}
Urinary tract infections and diabetes are two of the most important infectious and metabolic diseases all-around the world. Documented data showed that diabetes is one of the most important causative reasons of getting UTIs. Virulent and resistant strains of Escherichia coli are the most important causative agents for UTIs. The present investigation was carried to in order to evaluate the distribution of uropathogenic $E$. coli as well as virulence factors and antimicrobial resistance pattern of bacterial isolates of diabetic and non-diabetic patients suffered from UTIs. A total of 300 urine samples were collected from diabetic and non-diabetic patients suffered from UTIs. Samples were cultured and those that were positive were subjected to PCR and disk diffusion method. Prevalence of UPEC strains in diabetic and healthy patients were $65.38 \%$ and $36.47 \%$, respectively. Significant difference was seen for the prevalence of UPEC strains between diabetic and healthy male patients $(P<\mathbf{0 . 0 1})$. Older patients had the higher prevalence of E. coli $(\mathrm{P}<0.01)$. Fim, cnf1, papGIII and hlyA were the most commonly detected virulence factors. UPEC strains showed the highest levels of resistance against ampicillin, gentamicin, ciprofloxacin and trimethoprim-sulfamethoxazole. UPEC strains of diabetic patients were more virulent and resistant $(P<0.05)$. Prescription of ceftriaxone, aztreonam, nalidixic acid and imipenem can reduce the risk of UTIs in diabetic patients.
\end{abstract}

Key words: Uropathogenic Escherichia coli, Virulence factors, Antibiotic resistance, Diabetes.

Diabetes and urinary tract infections (UTIs) are two most prominent diseases allaround ${ }^{1}$. Diabetes is associated with an earlier onset and increased severity of UTIs, resulting in costly and debilitating complications. Several investigations showed the higher distribution of UTIs, urethritis, pyelonephritis, cystitis and

\footnotetext{
* To whom all correspondence should be addressed. Tel.: 00989155144073;

E-mail: f.emampour@gmail.com
}

bladder dysfunction in diabetic in compare with non diabetic patients ${ }^{1-3}$.

Documented data revealed that near to $50 \%$ of people have been affected by UTIs allaround the world ${ }^{4}$. Uropathogenic Escherichia coli (E. coli (UPEC)) strains are the most com-mon causes of UTIs ${ }^{5,6}$. Evaluating the potential virulence genes is required to assess the pathogenicity of UPEC strains in UTIs. Successful colonization, establishment, and ultimately leading to UTIs by UPEC strains is based on the ability to 
adhere to host surfaces such as mucous membranes, urinary epithelial or kidney tissue. The most important virulence factors in UPEC strains are hemolysin (hly), P fim-briae (papcytotoxic necrotizing factor 1 ( $c n f 1)$, ), a fimbrial adhesin $(a f a)$, and $\mathrm{S}$ fimbriae $(s f a)^{5-8}$. Successful colonization and invasion of UPEC in the urinary tract depends on the expression of mentioned virulence factors.

One of the most essential aspects for control of the UTIs is treatment. Treatment of diseases caused by this bacterium often requires antimicrobial therapy; however, antibiotic-resistant strains of E. coli bacterium cause more severe diseases for longer periods of time than their susceptible isolates. Several studies showed that antibiotic resistance in UPEC is increasing nowadays ${ }^{8-10}$.

Iran is one of the most important sites of the world which has a high prevalence of UTIs caused by UPEC strains and also diabetes ${ }^{8,11-13}$. Therefore, epidemiological researches should be done to find the exact pathogenicity and antimicrobial resistance properties of various strains of $E$. coli in the cases of UTIs. The current survey was carried out in order to determine the distribution of virulence factors and antimicrobial resistance properties of UPEC strains isolated from diabetic and non diabetic patients suffered from UTIs.

\section{MATERIALSAND METHODS}

\section{Samples and Escherichia coli identification}

This cross sectional study was performed from May to December 2015. A total of 300 urine samples were collected from male patients with UTIs. In the other hand, urine samples were collected from diabetic $(n=130)$ and healthy $(n=$ 170) male patients suffered from UTIs. Patients of our study had the various ranges of age including 20-30, 30-40, 40-50, 50-60 and older than 60 years old. All samples were collected from the hospitalized pediatrics of educational hospitals in Tehran, Iran. Midstream urine was collected in sterile condition to decrease potential bacterial, cellular and artifactual contamination. All samples were immediately transferred to the laboratory at $4^{\circ} \mathrm{C}$. Totally, $3 \mathrm{~mL}$ of each sample was blended with $225 \mathrm{~mL}$ of nutrient broth (Merck, Germany) for 2 min at normal speed, using a Stomacher lab blender and incubated at $37^{\circ} \mathrm{C}$ for $24 \mathrm{~h}$. One milliliter sample of the nutrient broth culture was mixed with $9 \mathrm{~mL}$ of MacConkey broth (Merck, Germany) and further incubated at $37^{\circ} \mathrm{C}$ for $24 \mathrm{~h}$. One loop of each tube was streaked on MacConkey agar (Merck, Germany). A typical purple colony of E. coli was streaked on Eosin Methylene Blue agar (EMB agar) plate (Merck, Germany) and incubated at $37^{\circ} \mathrm{C}$ for 24h. A metallic green colony from each plate with typical E. coli morphology was selected and examined by biochemical tests, including hydrogen sulfide, citrate, urease and indole.

\section{Antimicrobial Susceptibility Testing}

The antibiotic susceptibility patterns were determined using the disk diffusion method according to the Clinical and Laboratory Standards Institute (CLSI) guidelines (20). The following antimicrobials were tested: cefoxitin (FOX: 30 ìg), amoxicillin-clavulanic acid (AMC: 20/10 $\mu \mathrm{g}$ ), ceftriaxone (CRO: 30 ìg), gentamicin (GEN: 10 ìg), ampicillin (AMP: 10 ìg), nitrofurantoin (NIT, 300 $\mu \mathrm{g}$ ), ceftazidim (CAZ: 30 ìg), aztreonam (ATM: 30

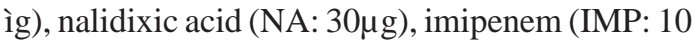
ìg), ciprofloxacin (CIP: 5 ìg) and trimethoprimsulfamethoxazole (SXT: 25 ìg). The quality control organism was E. coli ATCC 25922. Results were interpreted as susceptible or resistant according to criteria recommended by the CLSI and the manufacture protocols (Mast Companies, UK) (14). DNA extraction and $E$. coli identification

Bacterial strains were sub cultured overnight in Luria Bertani broth (Merck, Germany) and genomic DNA was extracted from typical colonies of E. coli using DNA extraction kit (Fermentas, Germany) according to manufacturer's instruction. All of the positive colonies were confirmed using the polymerase chain reaction (PCR) technique (15). PCR was performed with a total volume of $50 \mu \mathrm{L}$ including $2 \mathrm{mM} \mathrm{MgCl} 2,1 \mu \mathrm{M}$ of forward primer (5'-AGAGTTTGATC MTGGCTCAG-3'), $1 \mu \mathrm{M}$ of reverse primer (5'CCGTCAATTCATTTGAGTTT-3'), $5 \mu \mathrm{L}$ PCR buffer 10X, $200 \mu \mathrm{M}$ dNTP (Fermentas), 1 U Taq DNA polymerase (Fermentas) and $2.5 \mu \mathrm{L}$ DNA template. The DNA was then amplified by 31 successive cycles of denaturation at $95^{\circ} \mathrm{C}$ for $45 \mathrm{~s}$, primer annealing at $59^{\circ} \mathrm{C}$ for 60 s, and DNA chain extension at $72^{\circ} \mathrm{C}$ for 60 s.

Amplification of virulence factors

E. coli strains were cultured in LB broth 
at $37^{\circ} \mathrm{C}$ for 18 hours. Genomic DNA was extracted from the bacterial colonies using the DNA extraction kit (Fermentas, Germany) according to the manufacture's instruction. Table 1 shows the list of primers used for amplification of latent virulence factors ${ }^{8}$. All of the PCR reactions were done using the programmable thermocycler ( Mastercycler Gradiant Eppendorph, Germany). A PCR method was performed with a total volume of $50 \mu \mathrm{L}$ including $1.5 \mathrm{mM} \mathrm{MgCl} 2,0.4 \mu \mathrm{M}$ of forward primer, $0.4 \mu \mathrm{M}$ of reverse primer, $5 \mu \mathrm{L}$ PCR buffer $10 \mathrm{X}, 200$ $\mu \mathrm{M}$ dNTP (Fermentas), $1 \mathrm{U}$ Taq DNA polymerase (Fermentas), and $4 \mu \mathrm{L}$ DNA template. The DNA was then amplified by 30 suc cessive cycles of denaturation at $94^{\circ} \mathrm{C}$ for $60 \mathrm{~s}$, primer annealing at $63^{\circ} \mathrm{C}$ for $30 \mathrm{~s}$, and DNA chain extension at $72^{\circ} \mathrm{C}$ for 90 s with a programmable thermal cycler (Eppendorf, Mastercycler ${ }^{\circledR}$ 5330, Eppendorf-Netheler-Hinz GmbH, Hamburg, Germany). E. coli ATCC 25922 and sterile distilled water were used as positive and negative controls in all PCR reactions.

\section{Gel electrophoresis}

All PCR products were analyzed by electrophoresis (120 V/208 mA) in 1.5\% agarose gel and stained by ethidium bromide. A molecular weight marker with $100 \mathrm{bp}$ increments (100bp ladder, Fermentas, Germany) and 1 kbp increments (1000bp ladder, Fermentas, Germany) was used as size standard.

\section{Statistical analysis}

Data were analyzed using SPSS software (Version 17. SPSS Inc, United States) to find any significant correlation between incidences of virulence factors and antibiotics resistance pattern of uropathogenic $E$. coli isolated from diabetic and healthy males with urinary tract infection. Statistical significance was regarded at a $\mathrm{P}$ value $<$ 0.05 .

\section{RESULTS}

Results of the present investigation showed that $E$. coli strains had a high prevalence in diabetic and non diabetic patients suffered from UTIs. Table 2 represents the total distribution of Uropathogenic E.coli in diabetic and healthy patients of various age groups suffered from UTIs. We found that the total prevalence of E. coli in diabetic and healthy patients were $65.38 \%$ and $36.47 \%$, respectively. Significant statistical difference was seen for the prevalence of $E$. coli between diabetic and healthy male patients ( $P$ $<0.01$ ). The most commonly infected groups were older than 60 and 50-60 years old patients.

Table 1. List of primers used for amplification of latent virulence factors in the Escherichia coli strains of diabetic and healthy males suffered from urinary tract infections

\begin{tabular}{|c|c|c|}
\hline $\begin{array}{l}\text { Target } \\
\text { gene }\end{array}$ & Primer sequence (5'-3') & $\begin{array}{c}\text { Size of } \\
\text { product (bp) }\end{array}$ \\
\hline \multirow[t]{2}{*}{ papGI } & TCGTGCTGAGGTCCGGAATTT & 461 \\
\hline & TGGCATCCCCCAACATTATCG & \\
\hline \multirow[t]{2}{*}{ papGII } & GGGATGAGCGGGCCTTTGAT & 190 \\
\hline & CGGGCCCCCAAGTAACTCG & \\
\hline \multirow[t]{2}{*}{ papGIII } & GGCCTGCAATGGATTTACCTGG & 258 \\
\hline & CCACCAAATGACCATGCCAGAC & \\
\hline \multirow[t]{2}{*}{ fim } & GAGAAGAGGTTTGATTTAACTTATTG & 559 \\
\hline & AGAGCCGCTGTAGAACTGAGG & \\
\hline \multirow[t]{2}{*}{ afa } & GCTGGGCAGCAAACTGATAACTCTC & 750 \\
\hline & CATCAAGCTGTTTGTTCGTCCGCCG & \\
\hline \multirow[t]{2}{*}{ sfa } & CTCCGGAGAACTGGGTGCATCTTAC & 410 \\
\hline & CGGAGGAGTAATTACAAACCTGGCA & \\
\hline \multirow[t]{2}{*}{ hlyA } & AACAAGGATAAGCACTGTTCTGGCT & 1177 \\
\hline & ACCATATAAGCGGTCATTCCCGTCA & \\
\hline \multirow[t]{2}{*}{ cnf1 } & AAGATGGAGTTTCCTATGCAGGAG & 498 \\
\hline & TGGAGTTTCCTATGCAGGAG & \\
\hline
\end{tabular}


Table 2. Total distribution of Uropathogenic Escherichia coli in diabetic and healthy patients of various age groups suffered from UTIs

\begin{tabular}{lcccc}
\hline Types of samples & & No. samples & E. coli positive by culture (\%) & PCR confirmation (\%) \\
\hline Diabetic patients & $20-30$ & 21 & $7(33.33)$ & $7(33.33)$ \\
& $30-40$ & 23 & $10(43.47)$ & $10(43.47)$ \\
& $40-50$ & 20 & $12(60)$ & $12(60)$ \\
& $50-60$ & 22 & $16(72.72)$ & $16(72.72)$ \\
Healthy patients & $60<$ & 46 & $40(86.95)$ & $40(86.95)$ \\
& Total & 130 & $85(65.38)$ & $85(65.38)$ \\
& $20-30$ & 20 & $4(20)$ & $4(20)$ \\
& $30-40$ & 28 & $9(32.14)$ & $11(34.37)$ \\
& $40-50$ & 32 & $11(34.37)$ & $14(37.83)$ \\
& $50-60$ & 37 & $14(37.83)$ & $24(45.28)$ \\
& $60<$ & 53 & $24(45.28)$ & $62(36.47)$ \\
\hline
\end{tabular}

Table 3. Total distribution of Uropathogenic virulence factors in the Escherichia coli strains of diabetic and healthy patients suffered from UTIs

\begin{tabular}{lcccccccc}
\hline \multirow{2}{*}{$\begin{array}{l}\text { Types of } \\
\text { samples (No. positive) }\end{array}$} & \multicolumn{7}{c}{ Distribution of virulence factors (\%) } \\
\cline { 2 - 9 } & papGI & papGII & papGIII & fim & cnf1 & afa & sfa & hlyA \\
\hline $\begin{array}{l}\text { Diabetic patients } \\
(85)\end{array}$ & 13 & $17(20)$ & 80 & 82 & 80 & 74 & 78 & 79 \\
$\begin{array}{l}\text { Healthy patents } \\
(62)\end{array}$ & $(15.29)$ & & $(94.11)$ & $(96.47)$ & $(94.11)$ & $(87.05)$ & $(91.76)$ & $(92.94)$ \\
& $(14.51)$ & $(20.96)$ & $(91.93)$ & $(93.54)$ & $(91.93)$ & $(79.03)$ & $(87.09)$ & $(91.93)$
\end{tabular}

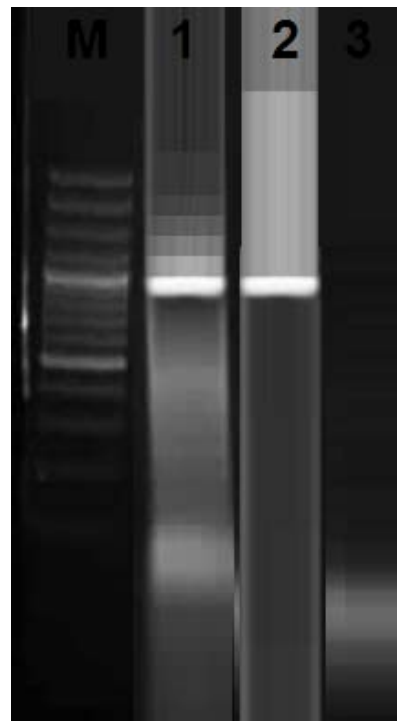

Fig. 1. Results of the gel electrophoresis for confirmation of Escherichia coli in the genomic DNA extracted from the bacterial colonies. M: $100 \mathrm{bp}$ ladder, 1: positive samples for the 16SrRNA gene (919 bp), 2: Positive control and 3: negative control
Statistically significant difference was seen for the prevalence of $E$. coli between various age groups $(\mathrm{P}<0.01)$

Table 3 shows the total distribution of Uropathogenic virulence factors in the $E$. coli strains of diabetic and healthy patients suffered from UTIs. The most commonly detected virulence factors in the UPEC strains of the diabetic patients were fim (96.47\%), cnf1 (94.11\%), papGIII (94.11\%) and hlyA (92.94\%). Those of non-diabetic patients were fim (93.54\%), hlyA (91.93\%) and cnf1 (91.93\%). Statistically significant differences were seen between the prevalence of various virulence factors $(\mathrm{P}<0.05)$.

Table 4 represents the antibiotic resistance pattern of Uropathogenic E. coli strains of diabetic and healthy patients suffered from UTIs. E. coli strains harbored the highest levels of resistance against ampicillin, gentamicin, ciprofloxacin and trimethoprim-sulfamethoxazole. This finding in the diabetic patients was higher than healthy ones but there were no significant differences. 


\section{DISCUSSION}

The results of te present investigation revealed that diabetic patients and especially diabetic males are more prone to get UTIs tan non diabetic patients. Total prevalence of UPEC strains in diabetic and non diabetic patients were $65.38 \%$ and $36.47 \%$, respectively. Higer prevalence of resistant and virulent strains of $E$. coli in diabetic patients was another important finding of our study.

Higher prevalence of virulent and resistant UPEC strains in diabetic than non diabetic patients is due to the fact that diabetes is a causative agent for the suppression of level of immunity in human. In this situation, occurrence of infections like UPEC strains has been increased. The mechanisms of diabetes caused them to more sensitive to various types of infections. Decrease in the level of immunity caused to occurrence of higher resistant of bacterial strains against commonly used antibiotics. Besides, lack of powerful immunity caused bacterial strains to produce more important secretary and non secretary virulence factors.

To our best knowledge the frequency of epidemiological investigations in this field is so scarce. In a study which was conducted in order to investigate the prevalence of virulence factors and phylogenetic characterization of uropathogenic E. coli causing urinary tract infection in patients with and without diabetes mellitus ${ }^{16}$, results showed that there was no significant difference in distribution of virulence factors of UPEC causing UTI from patients with and without diabetes. PapC gene was most prevalent in both groups of patients, followed by hly gene which was similar to our findings. Only cnf-1 gene was observed to be significantly associated $(\mathrm{p}<0.05)$ with the non-diabetic status than diabetic. Bangal investigation ${ }^{17}$ showed that E. coli was the most prevalent cause of UTIs in diabetic patients. Amikacin exhibited only 3\% resistance and gentamicin exhibited 26.9\% resistance with E. coli. Prevalence of resistance against nitrofurantoin was low. Besides, resistance against cefixime and ceftriaxone was moderate and amoxicillin and ciprofloxacin showed the highest resistances in all these cases which wall were similar to our results. In a study which was conducted by Hamdan et al. (2015) ${ }^{18}$, the predominant isolates 
were E. coli (56.4\%). Six, four, three, and two of 22 E. coli isolates showed resistance to ampicillin, co-trimoxazole, nitrofurantoin, and amoxicillinclavulanic acid, respectively. In addition, all $22 \mathrm{E}$. coli isolates were sensitive (100\%) to gentamicin and cephalexin.

Our result represents that the prevalence of UPEC resistance against amoxicillin-clavulanic acid gentamicin, ampicillin, ciprofloxacin and trimethoprim-sulfamethoxazole were more than $60 \%$ which was considerable high. Irregular and excessive administration of antibiotics is the most important reason for the high prevalence of resistance against commonly used antimicrobial agents in our study. In fact, Medical practitioners don't use from rapid and simples methods like disk diffusion technique to evaluate the exact profile of antibiotic resistance in the cases of UTIs especially those caused by UPEC strains. Therefore, antibiotic resistance will occur in a short period of time. Differences in the levels of antibiotic resistance which were showed in various studies maybe due to the differences in the availability of antibiotics, pattern of resistance, idea of medical practitioners to antibiotic administration and even cost of antibiotic agents in each zone and/or country.

We also found that the UPE3C strains harbored the high levels of virulence factors and especially fim, cnf1, papGIII and hlyA. Momtaz et al. (2013) ${ }^{8}$ represented that set1, fim, cnf1, papGIII and hlyA were the most commonly detected virulence factors in the UPEC strains of patients suffered from UTIs which was entirely similar to our findings. High prevalence of fim, hly, sfa, afa and also various types of pap genes were also reported by Dormanesh et al. (2014) $)^{15}$, Arabi et al. $(2012)^{19}$, Asadi Karam et al. (2012) ${ }^{20}$, Karimian et al. (2012) ${ }^{21}$, Harwalkar et al. (2013) $)^{22}$, Yun et al. (2014) ${ }^{23}$ and Zhao et al. (2009) ${ }^{24}$. These genes are mainly associated with adhesion, colonization and invasion of bacterial strains into the urinary epithelial cells.

\section{CONCLUSIONS}

In conclusions, we identified a large numbers of virulence factors and antimicrobial resistance properties in the UPEC strains isolated from diabetic and non diabetic patients suffered from UTIs. Higher numbers of UPEC strains, virulence factors and also antibiotic resistance pattern in the diabetic than non-diabetic patients are other important findings of our investigation. Older patients due to their lower levels of immunity had the highest prevalence of UPEC strains. The most commonly detected virulence genes are fim, cnf1, papGIII and $h l y A$ and UPEC strains harbored the highest levels of resistance against amoxicillinclavulanic acid gentamicin, ampicillin, ciprofloxacin and trimethoprim-sulfamethoxazole antimicrobial agents. We found that prescription of ceftriaxone, aztreonam, nalidixic acid and imipenem regarding the results of the disk diffusion can reduce the risk of UTIs in diabetic patients.

\section{REFERENCES}

1. Patterson JE, Andriole VT. Bacterial urinary tract infections in diabetes. Infect Dis Clin North Am. 1997;11(3):735-50.

2. Klouwens MJ, Blok WL, Witmer AN, Verouden CJ, Mura M. Serious complications of urinary tract infection in diabetes: emphysematous pyelonephritis and endogenous endophthalmitis. Ned Tijdschr Geneeskd. 2013; 157(7): A5243.

3. Lye WC, Chan RK, Lee EJ, Kumarasinghe G. Urinary tract infections in patients with diabetes mellitus. J Infect. 1992; 24(2):169-74.

4. Najar MS, Saldanha CL, Banday KA. Approach to urinary tract infections. Indian J Nephrol. 2009; 19(4):129-39.

5. Totsika M, Moriel DG, Idris A, Rogers BA, Wurpel DJ, Phan MD, Paterson DL, Schembri MA. Uropathogenic Escherichia coli mediated urinary tract infection. Curr Drug Targets. 2012; 13(11):1386-99.

6. Mazumdar K, Dutta NK, Dastidar SG, Motohashi N, Shirataki Y. Diclofenac in the management of E. coli urinary tract infections. In vivo. 2006; 20(5): 613-9.

7. Ejrnæs K. Bacterial characteristics of importance for recurrent urinary tract infections caused by Escherichia coli. Dan Med Bull. 2011; 58(4): B4187.

8. Momtaz H, Karimian A, Madani M, Safarpoor Dehkordi F, Ranjbar R, Sarshar M, Souod N. Uropathogenic Escherichia coli in Iran: serogroup distributions, virulence factors and antimicrobial resistance properties. Ann Clin Microbiol Antimicrob. 2013;12:8.

9. Okesola AO, Aroundegbe TI. Antibiotic resistance pattern of uropathogenic Escherichia 
coli in South West Nigeria. Afr J Med Med Sci. 2011; 40(3): 235-8.

10. Adib N, Ghanbarpour R, Solatzadeh H, Alizade $\mathrm{H}$. Antibiotic resistance profile and virulence genes of uropathogenic Escherichia coli isolates in relation to phylogeny. Trop Biomed. 2014; 31(1): 17-25.

11. Azimi-Nezhad M, Ghayour-Mobarhan M, Parizadeh MR, Safarian M, Esmaeili H, Parizadeh SM, Khodaee G, Hosseini J, Abasalti Z, Hassankhani B, Ferns G. Prevalence of type 2 diabetes mellitus in Iran and its relationship with gender, urbanisation, education, marital status and occupation. Singapore Med J. 2008; 49(7): 571-6.

12. Haghdoost AA, Rezazadeh-Kermani M, Sadghirad B, Baradaran HR. Prevalence of type 2 diabetes in the Islamic Republic of Iran: systematic review and meta-analysis. East Mediterr Health J. 2009; 15(3): 591-9.

13. Dehbanipour R, Rastaghi S, Sedighi M, Maleki N, Faghri J. High prevalence of multidrugresistance uropathogenic Escherichia coli strains, Isfahan, Iran. J Nat Sci Biol Med. 2016;7(1):226.

14. Clinical and Laboratory Standards Institute (CLSI):Performance Standards forAntimicrobial Disk Susceptibility Tests, Approved standardNinth Edition(M2-A9).Wayne, PA: Clinical and Laboratory Standards Institute; 2014.

15. Dormanesh B, Safarpoor Dehkordi F, Hosseini S, Momtaz H, Mirnejad R, Hoseini MJ, Yahaghi E, Tarhriz V, Khodaverdi Darian E. Virulence factors and o-serogroups profiles of uropathogenic Escherichia coli isolated from Iranian pediatric patients. Iran Red Crescent Med J. 2014; 16(2): e14627.

16. Harwalkar A, Gupta S, Rao A, Srinivasa H. Prevalence of virulence factors and phylogenetic characterization of uropathogenic Escherichia coli causing urinary tract infection in patients with and without diabetes mellitus. Trans $R$ Soc
Trop Med Hyg. 2015; 109(12): 769-74.

17. Shill MC, Huda NH, Moain FB, Karmakar UK. Prevalence of uropathogens in diabetic patients and their corresponding resistance pattern: results of a survey conducted at diagnostic centers in dhaka, Bangladesh. Oman Med J. 2010; 25(4): 282-5.

18. Hamdan HZ, Kubbara E, Adam AM, Hassan OS, Suliman SO, Adam I. Urinary tract infections and antimicrobial sensitivity among diabetic patients at Khartoum, Sudan. Ann Clin Microbiol Antimicrob. 2015; 14: 26.

19. Arabi S, Tohidi F, Naderi S, Nazemi A, Jafarpour M, Naghshbandi. The common fimbarie genotyping in Uropathogenic Escherichia coli. Ann Biol Res. 2012; 3(10): 4951-4954.

20. sadi Karam MR, Oloomi M, Habibi M, Bouzari S. Cloning of fimH and fliC and expression of the fusion protein FimH/FliC from Uropathoge Escherichia coli (UPEC) isolated in Iran. Iran J Microbiol, 2012; 4(2): 55-62.

21. Karimian A, Momtaz H, Mahbobe Madani M. Detection of uropathogeni Escherichia coli virulence factors in patients with urinary tract infection in Iran. Afr J Microbiol Res 2012; 6(39): 6811-6816.

22. Harwalkar A, Gupta S, Rao A, Srinivasa H. Prevalence of virulence factors and phylogenetic characterization of uropathogenic Escherichia coli causing urinary tract infection in patients with and without diabetes mellitus. Trans $R$ Soc Trop Med Hyg. 2015;109(12):769-74.

23. Yun KW, Kim HY, Park HK, Kim W, Lim IS. Virulence factors of uropathogenic Escherichia coli of urinary tract infections and asymptomatic bacteriuria in children. $J$ Microbiol Immunol Infect. 2014; 47(6):455-61.

24. Zhao L, Chen X, Zhu X, Yang W, Dong L, Xu $X$, Gao S, Liu X. Prevalence of virulence factors and antimicrobial resistance of uropathogenic Escherichia coli in Jiangsu province (China). Urology. 2009;74(3):702-7. 